This copy is for your personal, non-commercial use only.

If you wish to distribute this article to others, you can order high-quality copies for your colleagues, clients, or customers by clicking here.

Permission to republish or repurpose articles or portions of articles can be obtained by following the guidelines here.

The following resources related to this article are available online at www.sciencemag.org (this information is current as of August 28, 2013 ):

Updated information and services, including high-resolution figures, can be found in the online version of this article at:

http://www.sciencemag.org/content/285/5424/83.full.html

This article cites 8 articles, 3 of which can be accessed free:

http://www.sciencemag.org/content/285/5424/83.full.html\#ref-list-1

This article has been cited by 332 article(s) on the ISI Web of Science

This article has been cited by 20 articles hosted by HighWire Press; see:

http://www.sciencemag.org/content/285/5424/83.full.html\#related-urls

This article appears in the following subject collections:

Chemistry

http://www.sciencemag.org/cgi/collection/chemistry 


\title{
Microfabrication Inside Capillaries Using Multiphase Laminar Flow Patterning
}

\author{
Paul J. A. Kenis, Rustem F. Ismagilov, George M. Whitesides*
}

The reaction of species in solutions flowing laminarly (without turbulent mixing) inside capillaries was used as the basis for a broadly applicable method of microfabrication. In this method, patterning occurs as a result of transport of reactive species to interfaces within the capillary by laminar flow. A wide range of chemistries can be used to generate structures with feature sizes of less than 5 micrometers and with spatial localization to within 5 micrometers. The method is applicable to the patterning of metals, organic polymers, inorganic crystals, and ceramics on the inner walls of preformed capillaries, using both additive and subtractive processes.

The methods most commonly used to fabricate microstructures inside microchannels (that is, capillaries) are based on conventional photolithography. The capillary and its internal structures are built in a series of planar fabrication steps, with each step requiring a mask, and with both registration among layers and sealing between layers being important technical issues (1). In the method described here the capillary is formed first (a relatively straightforward task for capillary diameters of 50 to $400 \mu \mathrm{m})$. Then, multiple liquid streams flowing laminarly-that is, without turbulent mixing - at low Reynolds numbers $(R e)$ are combined, with the chemical reactions occurring either between the streams and the interior surface of the capillary, or at the interface between the streams, to generate the desired structures. Because these processes occur inside a preexisting capillary, no registration steps are required. Fabrication by this process (which we call "fabrication using laminar flow" or FLO) can generate a wide variety of structures and devices - metal wires and electrodes, regions in which $\mathrm{SiO}_{2}$ has been removed by etching from $\mathrm{Si} / \mathrm{SiO}_{2}$ wafers, ridges of organic polymer, lines of crystals - that are $<10 \mu \mathrm{m}$ wide, and can localize these structures within the capillary with an accuracy of $\sim 5 \mu \mathrm{m}$. We believe that it will be especially useful in fabricating structures for use in microanalytical, microsynthetic, microfluidic, and microelectromechanical systems (MEMS).

The Reynolds number, $R e=v l \rho / \mu$, characterizes the tendency of a flowing liquid phase to develop turbulence (2). The lower the velocity ( $v$, in meters per second) of the liquid flow, the diameter of the capillary $(l$, in

Department of Chemistry and Chemical Biology, Harvard University, 12 Oxford Street, Cambridge, MA 02138, USA.

*To whom correspondence should be addressed. Email: gwhitesides@gmwgroup.harvard.edu meters), and the density of the liquid ( $\rho$, in kilograms per cubic meter), and the higher the viscosity ( $\mu$, in kilograms per meter per second) of the liquid, the lower is Re. Laminar flow occurs in fluidic systems with $R e<$ $\sim 2000$, and turbulent flow in those with $R e>\sim 2000$. Two or more distinct fluid streams moving in the same capillary at low $R e$ do not develop turbulence at the interface between them, or at the interface with the capillary walls: the only mechanism of mixing of their components is diffusion across the former interface (Fig. 1A). We usually used high flow rates $(v \sim 0.50 \mathrm{~m} / \mathrm{s})$ to minimize diffusional broadening of the etched or deposited zone downstream from the point at which the separate flows joined (3). The microfluidic system consisted of a polydimethylsiloxane (PDMS) membrane, with channels molded in its surface, sealed against the flat surface of a glass slide or a PDMS block (4). In these systems, $v$ can be controlled with surface tension, gravity, the application of electrical potentials, or pressure (5).
The regions of capillary that we wished to pattern ranged from tens of micrometers to several centimeters in length. Because many combinations of parallel fluid streams can be generated by using "Y" or "T" junctions (or their extensions to multiple streams), it is possible to bring a wide variety of solutions in contact with one another and with the walls of a capillary, and to take advantage of the range of chemistries available in these combinations to deposit material onto (or etch material from) the inner walls of the capillary. For example, localized etching of gold was achieved by sealing an elastomeric membrane with a zigzag channel to a glass slide covered with a thin $(250 \AA)$, semitransparent layer of gold. Parallel laminar flow of water and an aqueous commercial gold etchant resulted in the selective removal of $\mathrm{Au}$ in one half of the channel (Fig. 1B). For electroless deposition of silver, the elastomeric membrane with a zigzag channel was sealed to a block of PDMS. Parallel laminar flow of the two components of a commercial electroless silver plating solution (6) in the channel resulted in deposition of metallic silver at the interface between the two phases (Fig. 1C). This silver "wire" was electrically continuous, significantly narrower than the channel (that is, FLO accomplished size reduction while patterning), and smoother than the walls of the channel (7) (that is, FLO also exhibited a form of error correction) (8).

FLO can be used to generate a variety of structures. Selective etching of $\mathrm{SiO}_{2}$ on a $\mathrm{Si}$ wafer that forms one of the walls of a capillary was achieved by generating HF at the interface between juxtaposed, aqueous laminar flows of $\mathrm{HCl}$ and $\mathrm{KF}$ (Fig. 2A). A polymeric structure was precipitated at the interface between two aqueous phases containing oppositely charged polymers flowing laminarly in parallel (Fig. 2B). Chemiluminescence (9) occurred inside a capillary at the
Fig. 1. (A) Optical micrograph of twophase laminar flow: two aqueous phases, each colored with a different dye (black ink and Congo Red) brought together with a " $Y$ " junction in an elastomeric microfluidic system (PDMS). Only diffusional mixing over distances of several centimeters is observed. (B) Optical micrograph of a pattern etched in Au in a zigzag channel (PDMS) that is sealed to an Aucovered glass slide. (C) A silver wire deposited in a zigzag channel at the laminar flow interface between solutions containing the components of an electroless silver plating solution.

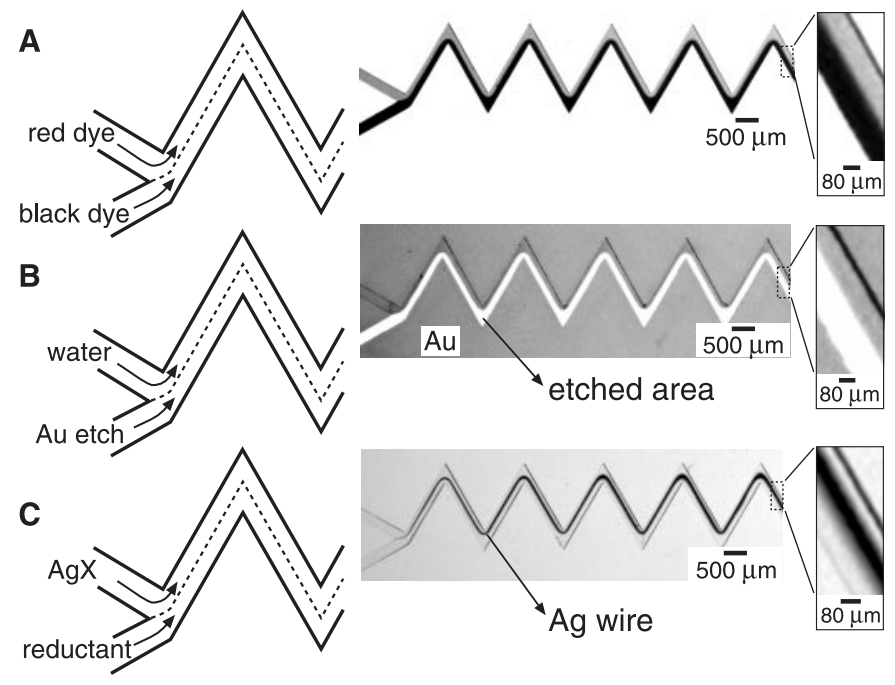


R E P O R T S

A

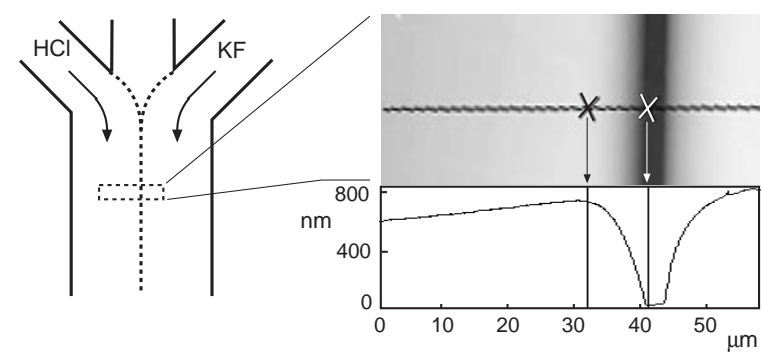

B
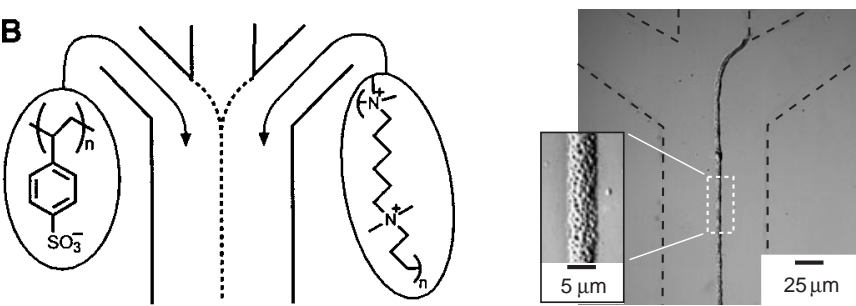

Fig. 2. (A) Atomic force microscopy profile of a trench (half-width $=6$ $\mu \mathrm{m}$ ) in $\mathrm{SiO}_{2}$ on a Si wafer etched by $\mathrm{HF}$ that is generated on the interface of laminar flow of $\mathrm{KF}\left(2 \mathrm{M}\right.$ in $\left.\mathrm{H}_{2} \mathrm{O}\right)$ and $\mathrm{HCl}\left(2 \mathrm{M}\right.$ in $\left.\mathrm{H}_{2} \mathrm{O}\right)$. (B) Optical micrograph of a polymeric structure deposited on glass at the laminar flow interface of $0.005 \%$ aqueous solutions of poly(sodium 4-styrenesulfonate) and hexadimethrine bromide. (C) Optical micrograph of
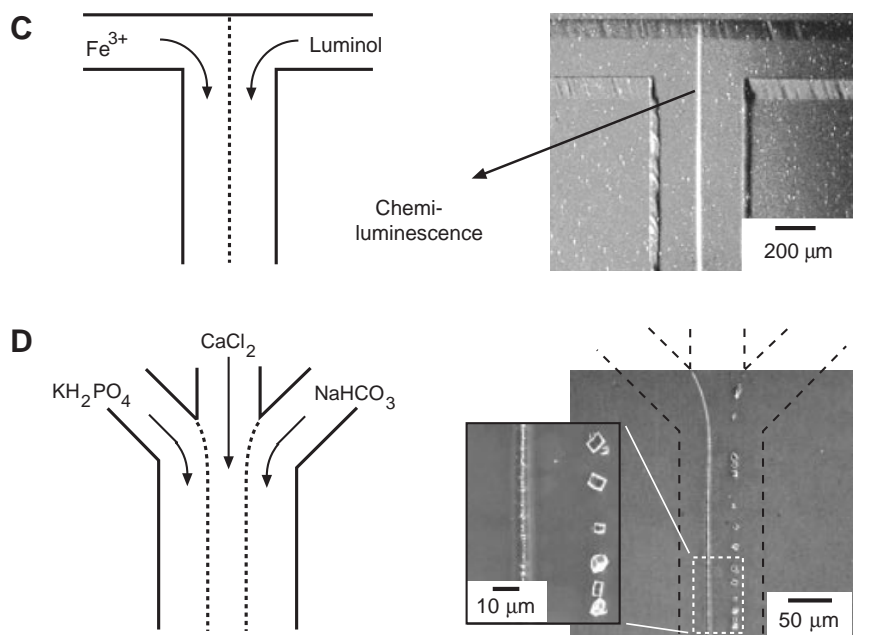

chemiluminescence at the interface of a two-phase aqueous laminar flow system: $\mathrm{K}_{3} \mathrm{Fe}^{\mathrm{III}}(\mathrm{CN})_{6}(0.1 \mathrm{M})$ and luminol $(0.05 \mathrm{M}$, in $0.1 \mathrm{M} \mathrm{NaOH})$ in the presence of $\mathrm{O}_{2}$. (D) Scanning electron microscopy images of calcite (right) and apatite (left) deposited simultaneously on a self-assembled monolayer at the interface of aqueous laminar flows of $\mathrm{NaHCO}_{3}\left(16 \mathrm{mM}\right.$ in $\mathrm{H}_{2} \mathrm{O}$, buffered to $\mathrm{pH} 8.5), \mathrm{CaCl}_{2}(25 \mathrm{mM})$, and $\mathrm{KH}_{2} \mathrm{PO}_{4}$ (3.6 mM, pH 7.4).
A
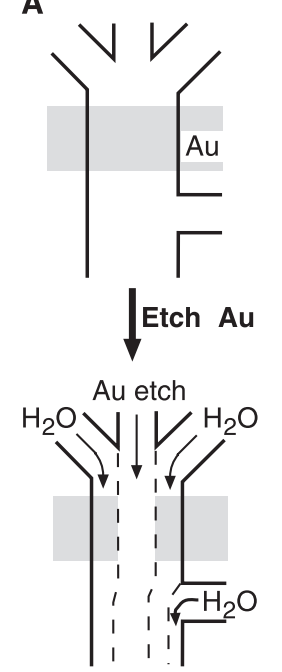

Deposit Ag
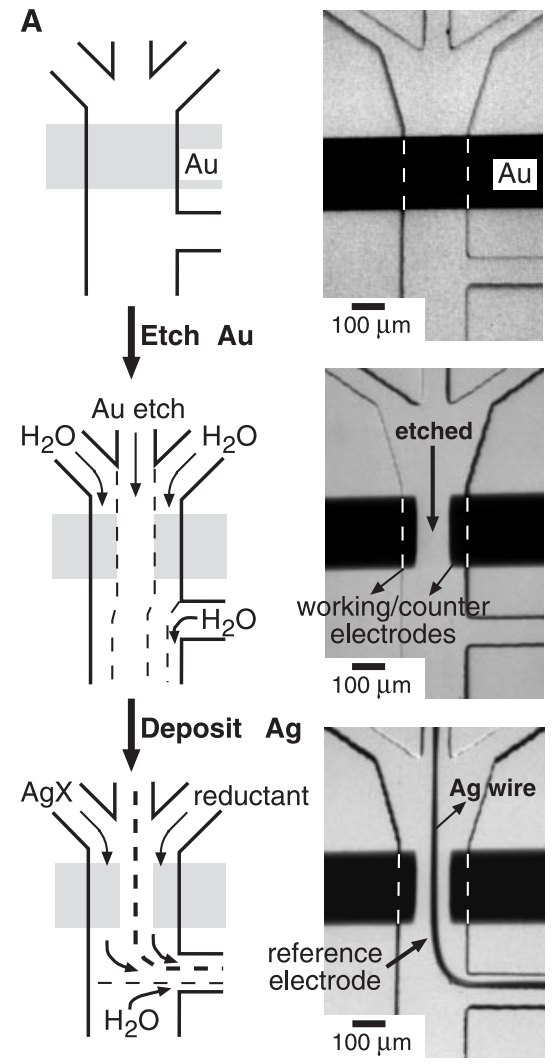
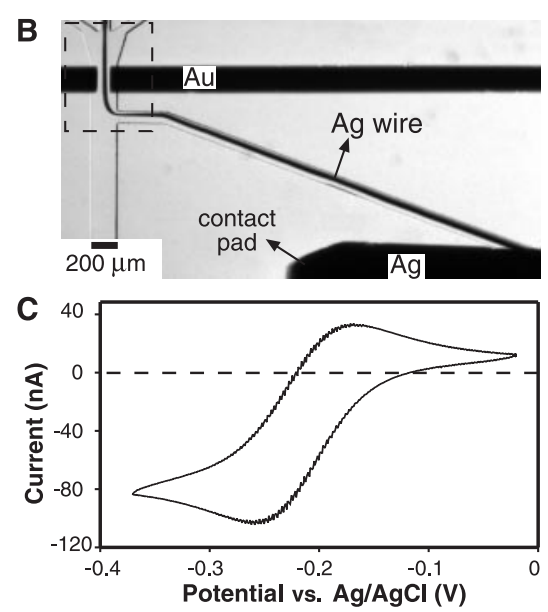

Fig. 3. (A) Optical micrographs of the stepwise fabrication of a three-electrode system inside a 200- $\mu \mathrm{m}$-wide channel. Two gold electrodes (counter and working) are formed by selectively etching the gold stripe that widens beyond the outer edge of the PDMS membrane into contact pads in the middle of the channel with a three-phase laminar flow system. A silver reference electrode is fabricated at the interface of a two-phase laminar flow (14). (B) Overview picture of the three-electrode system including the Ag contact pad. The dashed box corresponds to the last picture shown in (A). (C) Cyclic voltammogram of $\sim 5 \mathrm{nl}$ of $\mathrm{Ru}\left(\mathrm{NH}_{3}\right)_{6} \mathrm{Cl}_{3}$ in water $(2 \mathrm{mM}, 0.1 \mathrm{M} \mathrm{NaCl}$ electrolyte) as recorded with the three-electrode system (scan rate $=100 \mathrm{mV} / \mathrm{s}$ ).

interface between two aqueous phases containing luminol and $\mathrm{Fe}(\mathrm{III})$ (Fig. 2C) (10). Control of crystallization of calcium carbonate (calcite) and various calcium phosphates (apatites) has been extensively studied because of its biological relevance (11). A uniform array of calcite single crystals (12) and a thin $(<20 \mu \mathrm{m}$ thick), continuous line of apatite (13) were generated simultaneously at the two interfaces between parallel laminar flows of aqueous $\mathrm{NaHCO}_{3}, \mathrm{CaCl}_{2}$, and $\mathrm{KH}_{2} \mathrm{PO}_{4}$ solutions inside rectangular capillaries fabricated by placing a PDMS membrane (with channels embossed in its surface) over a substrate consisting of a self-assembled monolayer of $\mathrm{HS}\left(\mathrm{CH}_{2}\right)_{15} \mathrm{COOH}$ on $\mathrm{Au}$ (Fig.
2D). This experiment illustrates the capability of FLO to support several different reactions in the same capillary simultaneously.

We used FLO for the fabrication of a more complex, electrically functional device by making an array of three microelectrodes inside a 200- $\mu \mathrm{m}$-wide rectangular capillary (Fig. 3A). This capillary was assembled by placing a PDMS membrane that contained the channel network on a glass slide with the main channel oriented perpendicularly to a gold stripe that had been deposited by electron beam evaporation. A two-electrode system was first generated by flowing an aqueous gold etchant across the gold stripe as the middle phase of a threephase laminar flow system, with water as the adjacent phases. Controlling the relative volumes of the three liquid phases injected into the capillary controlled the width of the area of Au that was etched in the middle of the capillary. To prevent etching of the Ag contact pad at the end of the smaller exit outlet (Fig. 3B), we applied a counter flow of water from this channel. The third, reference electrode was generated by depositing a silver wire at the interface of the two phases containing components of electroless silver plating solution, followed by treatment with $1 \% \mathrm{HCl}$ to form $\mathrm{AgCl}$ on the surface of the wire (14). The wire was directed into the smaller outlet toward the Ag contact pad (Fig. 3B) by applying a flow of water from the main outlet. We tested the performance of the final device using cyclic voltammetry (Fig. 3C). The volume required to fill the electrochemical active area was less than $5 \mathrm{nl}$, and therefore less than $10 \mathrm{pmol}$ of $\mathrm{Ru}\left(\mathrm{NH}_{3}\right)_{6} \mathrm{Cl}_{3}(5 \mathrm{nl}$ of $2 \mathrm{mM}$ solution) was used for electrochemical analysis. 
By taking advantage of the fact that parallel streams of fluid flowing at low Reynolds number can maintain sharp boundaries (on the scale of micrometers) over distances of several centimeters, that reactions can be localized at the interfaces (fluid-capillary and fluid-fluid-capillary) with high spatial precision, and that reactive species present in these streams can be transported or generated with great versatility by using appropriate chemistries, FLO provides a method of microfabrication inside capillaries and other small enclosed spaces. With techniques developed for rapid prototyping of microstructures in polymers (4), it is straightforward to fabricate the network of capillaries that is required to bring together a range of solutions, in an appropriate order, to carry out multistep fabrication. Fluid flow can be controlled with whichever method seems most convenient for the application at hand (applied pressure, surface tension, gravity, or applied electrical potential). The liquid-liquid interfaces, and thus the features that are fabricated, can be positioned to within a few percent of the channel width by controlling the relative volumes of the different liquid streams entering the region in which reaction occurs. This procedure replaces the multiple stages of photolithography and pattern registration involved in photolithographic fabrication with a physical process-laminar flow - and permits the use of a wide range of chemistries in patterning.

FLO is probably most useful for making small numbers of systems for laboratory use and is not presently suited for high-volume manufacturing. The types of laminar flow patterns that are accessible limit the patterns that can be generated, but for certain applications - especially inside the channels used in electrochemical, microanalytical, and microsynthetic system - these patterns will allow the fabrication of a variety of useful structures.

\section{References and Notes}

1. M. Madou, Fundamentals of Microfabrication (CRC Press, New York, 1997).

2. O. Reynolds, Philos. Trans. 174, 935 (1883); ibid. 186, 123 (1895).

3. At a flow rate of $0.50 \mathrm{~m} / \mathrm{s}, R e=50$ for aqueous phases in a 100- $\mu \mathrm{m}$-wide channel. The broadening of a liquid-liquid interface by diffusion is given by $\left\langle x_{\text {diff }}{ }^{2}\right\rangle$ $=2 D t_{\text {diff }}$ with diffusion coefficient $D\left(\mathrm{~cm}^{2} / \mathrm{s}\right)$ and $x_{\text {diff }}$ $(\mathrm{cm})$ and $t_{\text {diff }}(\mathrm{s})$ the distance and time of diffusion, respectively [P. W. Atkins, Physical Chemistry (Freeman, New York, 1994)]. The broadening of the features fabricated by FLO cannot be calculated with that equation alone because the profile of pressuredriven flow is parabolic [J. P. Brody, P. Yager, R. E. Goldstein, R. H. Austin, Biophys. J. 71, 3430 (1996)]. The chemical reaction with the surface occurs in the stationary boundary layer; therefore the correct equation should account for lateral diffusion of the reagent within the layer, diffusional exchange with the moving liquid above the layer, and depletion of the reagent by the reaction. Preliminary experiments showed that broadening of an etched Au area in- creases roughly as the square root of the distance from the point where the separate flows join. The formula given above predicts a similar dependence, indicating that diffusional exchange between the boundary layer and the moving liquid dominates under our conditions.

4. D. C. Duffy, J. C. McDonald, O. J. A. Schueller, G. M. Whitesides, Anal. Chem. 70, 4974 (1998).

5. We used pressure-driven flow generated with a syringe pump (Harvard Apparatus 22). Syringes were connected to the inlets of the microfluidic system by polyethylene tubing (Intramedic, inner diameter of $0.38 \mathrm{~mm}$ ).

6. HE-300 solutions, Peacock Laboratories, 1901 South 54 Street, Philadelphia, PA 19143, USA. The counterion of the silver salt ( $X$ in the figures) is proprietary.

7. The roughness of the channel walls is typically 5 to $10 \mu \mathrm{m}$; the edge roughness of the etched or deposited structures is usually less than $1 \mu \mathrm{m}$.

8. Similarly, we deposited silver on one half of the inner surface of capillaries (PDMS, glass) by parallel laminar flow of water and an aqueous phase containing the premixed components of the electroless silver plating solution.

9. M. J. Cormier, D. M. Hercules, J. Lee, Eds., Chemiluminescence and Bioluminescence (Plenum, New York, 1973).

10. The use of laminar flow for "microfluidic diffusion-

based separation and detection" has been described [B. H. Weigl and P. Yager, Science 283, 346 (1999)].

11. H. A. Lowenstam and S. Weiner, On Biomineralization (Oxford Univ. Press, Oxford, 1989); S. Mann, J. Webb, R. J. P. Williams, Eds., Biomineralization. Chemical and Biological Perspectives (VCH, Weinheim, Germany, 1989).

12. J. Aizenberg, A. J. Black, G. M. Whitesides, Nature 394, 868 (1998); J. Aizenberg, A. J. Black, G. M. Whitesides, ibid. 398, 495 (1999).

13. D. Walsh, J. D. Hopwood, S. Mann, Science 264, 1576 (1994); D. Walsh and S. Mann, Chem. Mater. 8, 1944 (1996).

14. The Ag wire extended into the middle inlet as a result of back flow during the first few seconds of the deposition process.

15. This work was financially supported by the Defense Advanced Research Projects Agency and NSF grant ECS-9729405. Materials Research Science and Engineering Center-shared facilities supported by the NSF under grant DMR-9400396 were used. P.J.A.K. acknowledges the Netherlands Organization for Scientific Research (NWO) for a postdoctoral fellowship. We acknowledge W. Huck and Y. Lu for their help with the atomic force microscopy and scanning electron microscopy experiments.

9 February 1999; accepted 15 April 1999

\title{
Shock Melting of the Canyon Diablo Impactor: Constraints from Nickel-59 Contents and Numerical Modeling
}

\author{
C. Schnabel, ${ }^{1}$ E. Pierazzo, ${ }^{2 *}$ S. Xue, ${ }^{3}$ G. F. Herzog, ${ }^{1 *}$ J. Masarik, ${ }^{4}$ \\ R. G. Cresswell, ${ }^{5}$ M. L. di Tada, ${ }^{5}$ K. Liu, ${ }^{5}$ L. K. Fifield ${ }^{5}$
}

Two main types of material survive from the Canyon Diablo impactor, which produced Meteor Crater in Arizona: iron meteorites, which did not melt during the impact; and spheroids, which did. Ultrasensitive measurements using accelerator mass spectrometry show that the meteorites contain about seven times as much nickel-59 as the spheroids. Lower average nickel-59 contents in the spheroids indicate that they typically came from 0.5 to 1 meter deeper in the impactor than did the meteorites. Numerical modeling for an impact velocity of 20 kilometers per second shows that a shell 1.5 to 2 meters thick, corresponding to 16 percent of the projectile volume, remained solid on the rear surface; that most of the projectile melted; and that little, if any, vaporized.

About 50,000 years ago, the impact of an iron meteoroid excavated Meteor Crater, Arizona $(1,2)$. During atmospheric entry and impact, some of the impactor remained solid, producing the Canyon Diablo meteorites. Most of the meteorites came from within $1.8 \mathrm{~m}$ of the

${ }^{1}$ Department of Chemistry, Rutgers University, Piscataway, NJ 08854, USA. ²unar and Planetary Laboratory, University of Arizona, Tucson, AZ 87521, USA. ${ }^{3}$ Graduate School of Oceanography, Narragansett Bay Campus, University of Rhode Island, Narragansett, RI 02882, USA. ${ }^{4}$ Space Sciences Laboratory, University of California, Berkeley, CA 94720, USA. ${ }^{5}$ Department of Nuclear Physics, Research School of Physical Sciences and Engineering, Australian National University, Canberra, ACT 0200, Australia.

*To whom correspondence should be addressed. E-mail: betty@lpl.arizona.edu; herzog@rutchem. rutgers.edu preatmospheric surface of the impactor (3, $4)$. Another portion of the impactor melted $(5,6)$, producing the millimeter-size spheroids found in the soils around Meteor Crater (7). Nininger (7) estimated the total spheroid inventory to be 4000 to 7500 metric tons, or about $5 \%$ of the total mass of the Canyon Diablo meteoroid in space $(1,8)$. Here, we deduce the original depth in the impactor of the material that melted to form the spheroids and compare the result with the predictions of computer simulations of the Canyon Diablo impact. The results provide new information about what happens to medium-size meteoroids when they strike Earth or other solid objects in the solar system. The results also lend support to the evidentiary basis for applying theories of cratering dynamics to the 